\title{
Accurate Site Occupancies for the O-atoms Diffused into the Annealed ZnSe Lattice
}

\author{
Z.K. Heiba and Karimat El-Sayed \\ Physics department, Faculty of science, Ain Shams University, \\ Cairo, Egypt. \\ The structural problem of oxygen diffusion into the ZnSe lattice \\ annealed in air at different temperatures was studied. Different possible sites \\ were tried for oxygen accommodation. It is found that oxygen atoms diffuse \\ into the vacant sites of the Se atoms formed due to Se evaporation. The relative \\ occupancy of the diffused oxygen was determined as a function of annealing \\ temperature. $\mathrm{ZnSeO} 3$ and $\mathrm{ZnO}$ phases start to develop at a temperature of 623 \\ $K$ and a period of 30 hours annealing time.
}




\section{Introduction}

Zinc chalcogenides such as $\mathrm{ZnSe}, \mathrm{ZnS}$ and $\mathrm{ZnTe}$ have an increasing applications in optoelectronic and optical devices for their effective use in dielectric mirrors, solar cells, blue light-emitting diodes, ...etc. Heat treatment of such chalcogenides may change the properties of the material. $\mathrm{ZnSe}$ undergoes marked oxidation at high temperatures and a lot of work studied the oxidation process in air [1-6]. The qualitative composition of the oxidation products was determined together with the microstructure properties of the annealed $\mathrm{ZnSe}$ [7]. Although gas adsorption plays an important role in the electrophysical properties of chalcogenides [8], it has not been given a considerable attention. Oxygen adsorption was reported as the main cause for the discrepancies in the electrical and optical properties of the air exposed and the as-grown (unexposed) PbTe thin films[6,8,9]. Similar discrepancies were found in the optical and photoconducting properties of $\mathrm{CdS}$ [10], $\mathrm{CdS}_{\mathrm{x}} \mathrm{Se}_{1-\mathrm{x}}$ [11] ,CdSe [12] and ZnSe [13] annealed in air. The relative occupancy of the oxygen atoms diffused into interstitial holes in the PbTe thin films was determined by Heiba and El-Sayed [14] at different annealing temperatures. The present work is aimed to determine the sites in the lattice into which the oxygen atoms are diffused, and the oxygen occupancy as a function of annealing temperature.

\section{Experimental and Analytical Procedure}

A $\mathrm{ZnSe}$ powder sample of certified reagent grade was annealed in air at the temperatures $373,473,523,623,673 \mathrm{~K}$. The sample was put in a pyrex tube and heated at the specified temperature for a period of 26-30 hours, then let to cool slowly to room temperature inside the furnace. X-ray diffraction data were collected with a Philips diffractometer model X'pert applying the step scanning mode in the rang $20-103^{\circ}$ with a step of $0.03^{\circ}(2 \theta)$ and a counting time of $3 \mathrm{~s}$ for each step. $\mathrm{Cu}-K_{\alpha}$ radiation was used with a graphite monochromator in the diffracted beam. Least-squares structure refinements were carried out using the FullProf program [15] based on Rietveld method. To obtain a physically significant information on the microstructure, the Pseudo-Voigt function, proposed by Thompson-Cox-Hastings [16], is used as a profile shape function. Anisotropic temperature factors were applied taking into consideration the restrictions imposed by the space group symmetry, so only the factors $B_{11}, B_{22}$ and $B_{33}$ were used. The quality of the least-squares structure refinement is defined by the Bragg-R-value given by:

$$
R_{B}=\sum_{j}\left|I_{j}(o b s)-I_{j}(c a l)\right| / \sum_{j}\left|I_{j}(o b s)\right|
$$


where $I_{j}$ is the Bragg intensity of the reflection $(\mathrm{j})$.

The goodness of profile fit was measured by the profile factor:

$$
R_{w p}=\left\{\sum_{i} W_{i}\left[Y_{i}(o b s)-Y_{i}(c a l)\right]^{2} / \sum_{i} W_{i} Y_{i}(o b s)^{2}\right\}^{\frac{1}{2}}
$$

where $W_{i}$ is a suitable weight taken as:

$$
W_{i}=1 / Y_{i}(\text { obs })
$$

and $Y_{i}$ is the intensity data point at the step (i).

The structural problem of oxygen diffusion into the lattice of a thermally heated $\mathrm{ZnSe}$ can be stated as follows: the ZnSe has a FCC structure with the space group $F \overline{4} 3 m$, where the Se occupies the 4-positions located at the tetrahedral sites $\frac{1}{4}, \frac{1}{4}, \frac{1}{4}+$ face center translation (F.C.), while $\mathrm{Zn}$ takes the 4positions located at $0,0,0+$ F.C. Two sets of 4 -interstitial holes are available for oxygen diffusion: octahedral holes located at $\frac{1}{2}, \frac{1}{2}, \frac{1}{2}+$ F.C. and tetrahedral holes located at $\frac{3}{4}, \frac{3}{4}, \frac{3}{4}+$ F.C. However, regarding the big difference between the radii of the octahedral and tetrahedral holes $\left(r_{T} / r_{O}=0.543\right.$, assuming a close packing), the two sets of holes can not normally be occupied by the same kind of atoms. On the other hand, heating of zinc selenide in air is accompanied by losses in weight, which is attributed to the evaporation of the highly volatile Se [4]. The vacancies created in the lattice due to Se sublimation provide another possible way for oxygen to diffuse into the lattice. A series of structural refinements were carried out to determine into which sites the oxygen may diffuse. The relative occupancy parameter for both $\mathrm{Zn}$ and $\mathrm{Se}$ is set free to be refined. Different trials were made to accommodate oxygen in octahedral, tetrahedral holes or in Se-vacancies. Relative occupancy of oxygen is set free to be refined, and in a subsequent refinement it is constrained with selenium occupancy. Isotropic crystallite size and microstrain are calculated from:

$$
\begin{aligned}
& \operatorname{size}(\text { cauchy })=360 x \lambda /\left(\pi^{2} \times Y\right) \\
& \operatorname{strain}(\text { gaussian })=(\pi / 1.8) x \sqrt{\left(U-U_{\text {ins }}\right)}
\end{aligned}
$$

where $(\mathrm{Y})$ is the particle size broadening and $(\mathrm{U})$ is the halfwidth-parameter of Cagliotti function. 


\section{Results and discussions}

Figure (1) shows the profile fitting resulting from the structure refinement of $\mathrm{ZnSe}$ annealed at $523 \mathrm{~K}$. For temperatures less than $623 \mathrm{~K}$, no peaks other than those of the $\mathrm{ZnSe}$ structure were observed. For annealing at temperatures of $623 \mathrm{~K}$ and more, three phases are identified; $\mathrm{ZnSe}, \mathrm{ZnO}$ and $\mathrm{ZnSeO}_{3}$, applying the Search-match program. Figure (2) shows the diffraction pattern as obtained for $\mathrm{ZnSe}$ annealed at $623 \mathrm{~K}$ for 30 hours. For a period of annealing of 2 hours, Stepanova et. al. [1] found that $\mathrm{ZnSeO}_{3}$ starts to develop at $723 \mathrm{~K}$. Nevertheless, increasing the period of annealing changes the character of oxidation and $\mathrm{ZnSeO}_{3}$ may develop at a lower

Table (1)

The structural parameters of $\mathrm{ZnSe}$ annealed at $573 \mathrm{~K}$.

\begin{tabular}{ccccccccc}
\hline Atom & position & $\mathrm{x}$ & $\mathrm{y}$ & $\mathrm{z}$ & $U_{11}\left(\AA^{2}\right)$ & $U_{22}\left(\AA^{2}\right)$ & $U_{33}\left(\AA^{2}\right)$ & occupancy \\
\hline $\mathrm{Zn}$ & $4 \mathrm{a}$ & 0 & 0 & 0 & 0.00876 & 0.00399 & 0.00399 & 0.0417 \\
& & & & & \pm .00059 & \pm .00058 & \pm .00059 & ------- \\
$\mathrm{Se}$ & $4 \mathrm{c}$ & $1 / 4$ & $1 / 4$ & $1 / 4$ & 0.00678 & 0.02271 & 0.02271 & 0.0291 \\
& & & & & \pm .00111 & \pm .00111 & \pm .00111 & \pm .0003 \\
& & & & & & & & \\
$\mathrm{O}$ & $4 \mathrm{c}$ & $1 / 4$ & $1 / 4$ & $1 / 4$ & 0.05874 & 0.01504 & 0.01504 & 0.0126 \\
& & & & & \pm .00111 & \pm .00111 & \pm .00111 & \pm .0003 \\
\hline
\end{tabular}

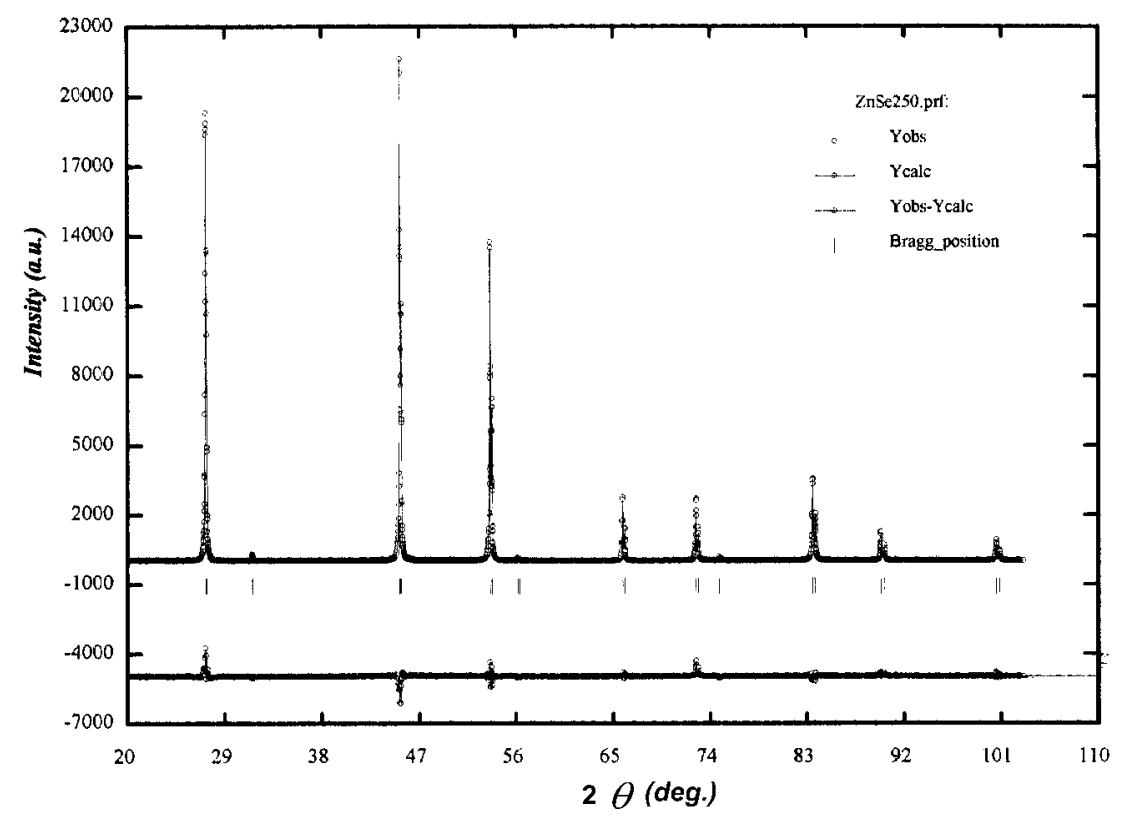

Fig. (1) : The profile fitting of $\mathrm{ZnSe}$ annealed at $523 \mathrm{~K}$ for 30 hours. 


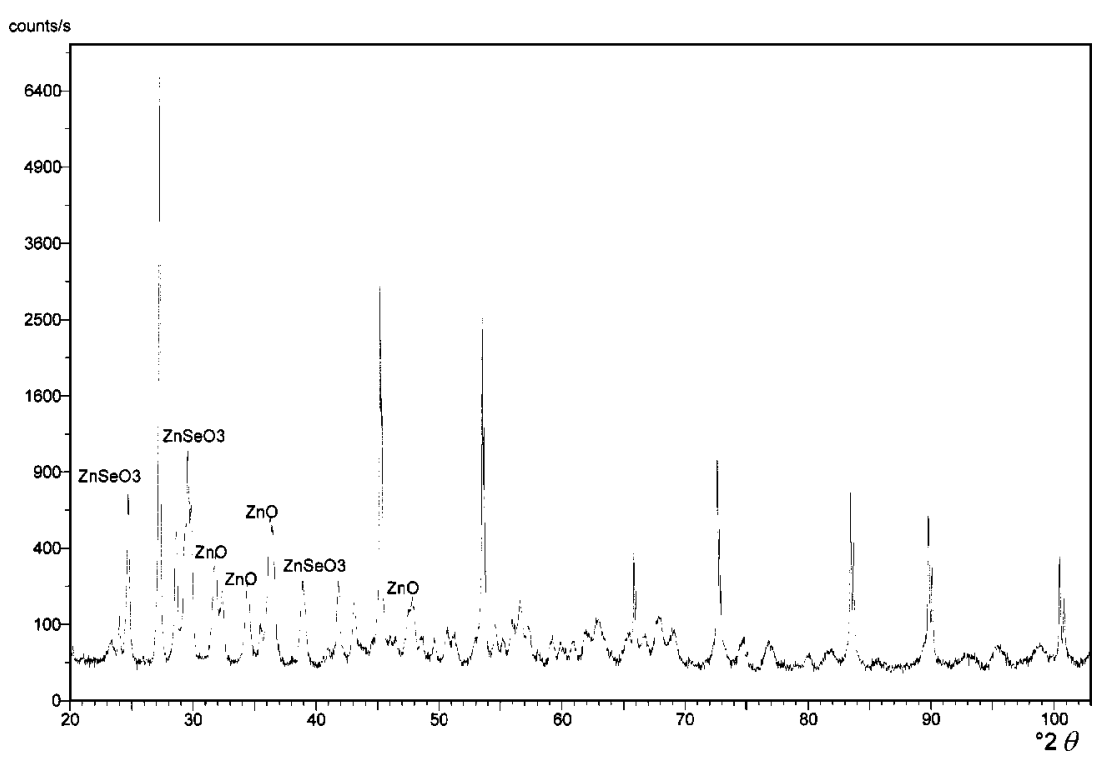

Fig. (2) : The diffraction pattern as obtained for $\mathrm{ZnSe}$ annealed at $673 \mathrm{~K}$, showing the $\mathrm{ZnSe}$ together with the growing oxide phases $(\mathrm{ZnO}$ and $\mathrm{ZnSeO} 3)$.

temperature[4]. The structural parameters obtained for $\mathrm{ZnSe}$ annealed at $573 \mathrm{~K}$ are given in Table (1). At the beginning of refinements, the anisotropic temperature factors of oxygen atoms were constrained to be equal to those of selenium, and after few cycles they left free to refine. Table (2) shows the lattice parameter (a), the average crystallite size and the relative occupancies of $\mathrm{Se}$ and $\mathrm{O}$ at different annealing temperatures. The relative occupancy of zinc atoms remains almost constant for all temperatures, while that of selenium atoms, more volatile, decreases with temperature.

Trials to accommodate oxygen atoms in the tetrahedral or octahedral holes gave high reliability factors and non-reasonable negative temperature factors. Moreover, some trials yielded occupancy values greater than the site occupancy. On the other hand, accommodating oxygen atoms in the vacant $\mathrm{Se}$ sites gave better reliability factors which means that the $4 \mathrm{c}$ Se-sites is more favorable than the octahedral and the other tetrahedral holes present in the lattice. As the annealing temperature is increased, the oxygen occupancy increases and that of selenium decreases. In subsequent refinements, the oxygen occupancy was unconstrained with that of selenium and a little bit lower occupancy was obtained for oxygen. Looking carefully at the results shown in table (2) one can see that the $\mathrm{R}_{\mathrm{B}}$ values of the samples annealed at 373 and 523 $K$ are $5.17 \%$ and $5.66 \%$ respectively, while the $R_{B}$ value of the sample annealed 
at $473 \mathrm{~K}$ is $8.16 \%$. This high reliability factor may be due to vacancies (point defects) left by the evaporated $\mathrm{Se}$ atoms at this temperature. The rate of oxidation at $473 \mathrm{~K}$ is less than the rate of Se evaporation, but by increasing the temperature at $523 \mathrm{~K}$ the rate of oxidation can compensate the rate of evaporation and accordingly most of the evaporated Se atoms are replaced by the oxygen atoms. This explanation can be also supported by the decrease in the averaged size at $473 \mathrm{~K}$ as shown in table (2).

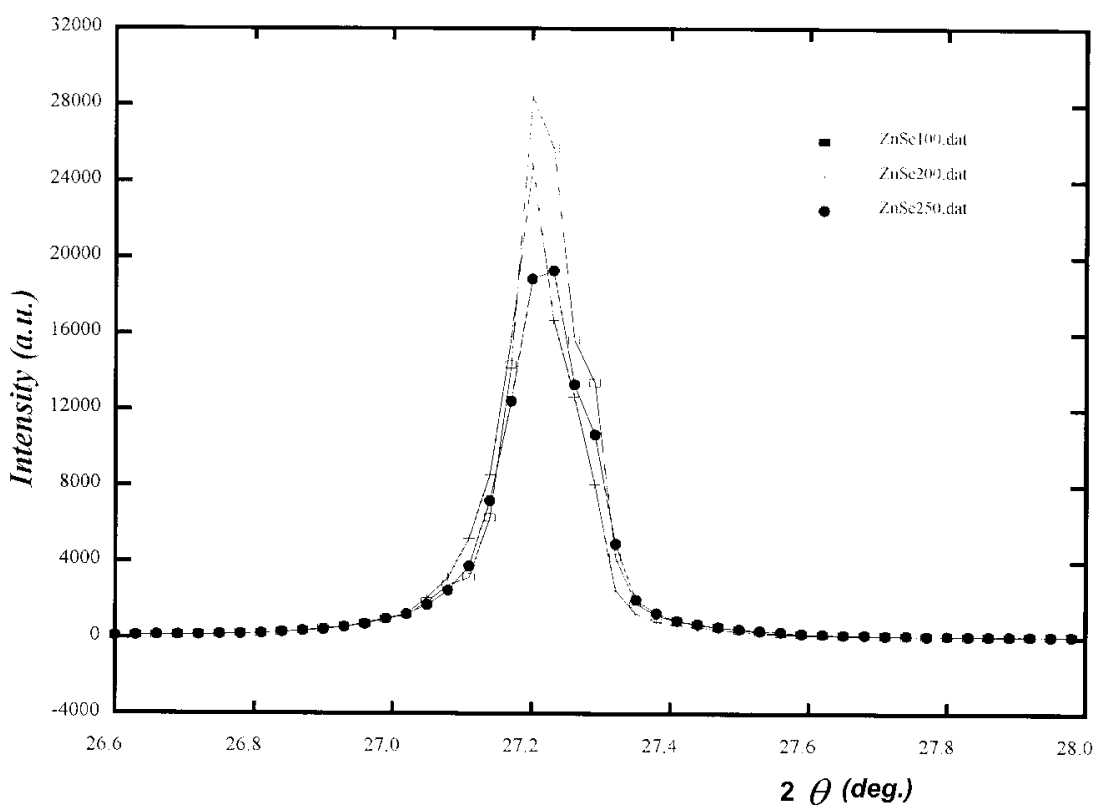

Fig. (3) : The (111) peak at different annealing temperatures.

Since the selenium vacancies and the oxygen sites are close packed normal to the [111] direction, a comparison between the intensities of the plane (111) obtained at different annealing temperatures could be a good indication for illustrating the oxygen diffusion into the lattice. Figure (3) shows the reduction in the intensity of the (111) plane due to the evaporation of selenium atoms as well as the diffusion of oxygen atoms.

The present results confirm the oxygen diffusion into the II-VI semiconductor compounds which was reported by many authors as the main cause of the unexpected 
Table (2)

The lattice parameter, the occupancy and the average size of $\mathrm{ZnSe}$ annealed at different temperatures.

\begin{tabular}{ccccccc}
\hline $\begin{array}{c}\text { Temp. } \\
(\mathrm{K})\end{array}$ & $\mathrm{a}(\AA)$ & \multicolumn{2}{c}{ Relative occupancy ${ }^{+}$} & $\begin{array}{c}\text { Strain } \\
\text { Se }\end{array}$ & $\begin{array}{c}\text { Average size* } \\
(\mathrm{nm})\end{array}$ & $R_{B} \%$ \\
\hline Room & 5.66965 & 0.0417 & 0.0000 & 0.11 & 181 & 5.11 \\
& \pm .00010 & \pm .000 & ----- & & & \\
373 & 5.66937 & 0.0362 & 0.0054 & 0.12 & 185 & 5.17 \\
& \pm .00013 & \pm .0013 & \pm .0013 & & & \\
473 & 5.66768 & 0.0329 & 0.0080 & 0.19 & 170 & 8.16 \\
& \pm .00013 & \pm .0014 & \pm .0014 & & & \\
523 & 5.66716 & 0.0291 & 0.0125 & 0.22 & 222 & 5.66 \\
& \pm .00011 & \pm .0004 & \pm .0004 & & & \\
\hline
\end{tabular}

* represents the volume averaged diameter of the crystallites.

+ site occupancy multipliers divided by the number of general positions of the space group.

discrepancies obtained in the electrical and optical properties $[8,10,11,12]$. The chemisorption of oxygen at the sample surface has a screening effect, while the oxygen incorporated into the lattice may give rise to a change in the band gap structure, and accordingly changes the physical properties. As seen from table (2), the relative occupancy of the oxygen incorporated into the lattice increases with the annealing temperature. The maximum occupancy will occur just before developing of $\mathrm{ZnSeO} 3$ or $\mathrm{ZnO}$ phases, which depends on both the annealing temperature and the duration of annealing time.

\section{References}

1. N.D. Stepanova, I.P. Kalinkin, and V.A. Sokolov, Izv. Akad. Nauk SSSR, Neorg. Mater., 11 (6), 1030, (1975).

2. M.P. Kulakov, and A.V. Fadeev, Izv. Akad. Nauk SSSR, Neorg. Mater., 19 (3), 347, (1983).

3. I.V. Korneeva and A.V. Novoselova, Zh. Neorb. Khim., 5 (11), 2265, (1960).

4. N.N. Gunchenko, G.N. Dronova, I.A. Maksimova, I.A. Mironova, V.N. Pavlova, and N.I. Pevtsova, Izv. Akad. Nauk SSSR, Neorg. Mater., 24 (1), 36, (1988). 
5. A.N. Polukarov and M.Zh. Makhmetove, Zh. Fiz. Khim., 46 (4), 1059 (1972).

6. Yu.A. Boikov and V.A.Kutasov, Sov. Phys. Solid State 28, 1 (1986).

7. A. Aly, "X-ray diffraction studies of oxygen implantation in $\mathrm{ZnSe}$ semiconductor material". M. Sc. thesis in physics, Ain shams university, faculty of science, Cairo, Egypt, (1993).

8. V. Damodara Das and K seetharama Bhat, J. Phys. D: Appl. Phys. 22, 162 (1989).

9. N.L.P. Sinha, B.K. Samantary, A.K. Chaudhuri and H.N. Bose, J. Phys. D: Appl. Phys 9, 795 (1976).

10. B.K. Gupta, O.P. Agnihotri and A. Raza, Thin solid films, 48, 153(1978).

11. O.P. Agnihotri and A.K. Raturi, Thin solid films 108, 313 (1983).

12. A.K. Raturi, R. ThangaraJ, A.K. Sharma, B.B. Tripathi and O.P. Agnihotri. Thin solid films, 91, 55 (1982).

13. Akio Kuroyanagi, J. Appl. Phys. 68, 5567 (1990).

14. Z. K. Heiba and Karimat El-Sayed, Egypt. J. Sol. 16, 65 (1991).

15. J. Rodriguez-Carvajal, Physica B 192, 55 (1993).

16. P. Thomson, D.E. Cox and J. B. Hastings, J. Appl. Cryst. 20, 79 (1987). 